\title{
EFFECTIVENESS OF ILIOTIBIAL BAND STRETCHING AND STRENGTHENING EXERCISES IN PATELLOFEMORAL PAIN SYNDROME
}

\author{
MALARVIZHI, NEHA BHATT \\ Department of , SRM College of Physiotherapy, SRM University, Chennai, Tamil Nadu, India. Email:
}

Received: 27 August 2016, Revised and Accepted: 08 November 2016

\begin{abstract}
Objective: The study objective was to find out the effectiveness of iliotibial band stretching and strengthening exercises in patellofemoral pain syndrome.

Methods: The study design was Quasi-experimental study design. 20 patients with patellofemoral pain syndrome were taken, with the age group of 18-40 years. 20 patients undergone pre- and post-test measurement of hip angle of adduction with the help of inclinometer and were given interferential therapy and iliotibial band stretching and strengthening exercises for 1-week duration. Outcome measures were inclinometer and visual analog scale (VAS).
\end{abstract}

Results: Statistical analysis was done using Paired t-test which showed a significant improvement in VAS score (2.57 \pm 1.09 ) and angle of hip adduction pre- and post-test $(-6.85 \pm 2.50)$.

Conclusion: This study concluded that 1 week of stretching and strengthening of iliotibial band exercises were effective in reducing pain and increasing hip adduction angle in patellofemoral pain syndrome.

Keywords: Iliotibial band, Interferential therapy, Test, Stretching and strengthening exercises, Inclinometer.

(C) 2017 The Authors. Published by Innovare Academic Sciences Pvt Ltd. This is an open access article under the CC BY license (http://creativecommons. org/licenses/by/4. 0/) DOI: http://dx.doi.org/10.22159/ajpcr.2017.v10i2.14914

\section{INTRODUCTION}

Patellofemoral pain syndrome is one of the most common musculoskeletal conditions in adolescents and young adults reported as occurring in $25 \%$ of general population. The symptoms most frequently reported are diffuse peripatellar and retropatellar pain, typically provoked by ascending or descending stairs, squatting, and sitting with flexed knees for a prolonged period of time. Patellofemoral pain syndrome is multifactorial in origin which includes malalignment of patella, excessive foot pronation, muscle imbalance, quadriceps insufficiency, patellar incongruence, excessive exercise, overtraining, tight lateral structures (iliotibial band), and ignorance of the condition [1].

Patellofemoral pain syndrome also known as anterior knee pain occurring in one in every four people. Those involved in athletics report an incidence $>25 \%$. The condition is more common in women than in men. Patellofemoral pain syndrome can be defined as retropatellar or peripatellar pain resulting from physical and biochemical changes in the patellofemoral joint. Patients with patellofemoral pain syndrome have anterior pain that typically occurs with activity and often worsens when they are descending steps or hills. It can also be triggered by prolong sitting. One or both knees can be affected [2].

The patella articulates with the patellofemoral groove in the femur. Several forces act on the patella to provide stability and keep it tracking properly. Patella moves up and down direction and it also tilts and rotates, so there are various points of contact between under surface of patella and femur, sometimes combined with maltracking of patella that is often not detectable is likely to be a mechanism of patellofemoral pain syndrome. The result is a presentation of retropatellar and peripatellar pain. The major contributing factors of patellofemoral pain syndrome are malalignment of lower extremity or patella, muscular imbalance of lower extremity, and overactivity [2].
The iliotibial band is a lateral thickening of the fascia lata that is composed of the distal fusion of the muscular fascia of the gluteus maximus, and tensor fascia lata muscle that originates at the iliac crest crosses the hip joint and extends distally to insert on the patella, tibia, biceps femoris, and vastus lateralis muscle and with the knee joint capsule. Along the lateral aspect of the knee, the iliotibial band overlies and blends with the lateral patellar retinaculum, which is an aponeurotic expansion of the vastus lateralis tendon. A tight iliotibial band has been implicated in several problems related to the knee including patellofemoral syndrome and iliotibial band friction syndrome. Several authors have suggested that tightness in the iliotibial band may contribute to patellofemoral syndrome and knee pain by pulling the patella laterally, thereby causing abnormal tracking of the patella in the trochlear groove. In patellofemoral pain syndrome, where a tight iliotibial band rubs over the lateral femoral epicondyle prominence with repetitive flexion and extension of the knee, has been reported as a relatively common condition in cyclists, ballet dancers, and runners, and has been attributed to the lack of flexibility of the iliotibial band [1]. Interferential therapy (IFT) is a pain relieving modality, in which two medium frequency currents are used to produce a low-frequency current. Medium frequency currents of around $4000 \mathrm{~Hz}$ are able to stimulate motor and sensory nerves, encounters a much lower skin impedance. The resistance of skin at $50 \mathrm{~Hz}$ is in the region of $3200 \Omega$, whereas with a frequency of $4000 \mathrm{~Hz}$, skin resistance is $40 \Omega$. The principle of IFT is to produce the interference effect where to produce medium frequency currents crosses in the patient's tissues. One of the current is of constant frequency $4000 \mathrm{~Hz}$, and other can be varied between $3900 \mathrm{~Hz}$ and $4000 \mathrm{~Hz}$. A beat frequency is created which is equal to the difference in the frequency between the two currents. IFT works on pain gait theory and blocks pain at different levels $[3,4]$.

Stretching is a general term used to describe any therapeutic maneuver designed to increase the mobility of soft tissues and subsequently improve the range of motion by elongating (lengthening) structures 
that have adaptively shortened and have become hypomobile over time [3]. Stretching is regarded as an integral part of fitness and supplied to a diverse population; it is very important to accurately define and prescribe the types of stretching modality that are most effective for achieving the common goals of increased flexibility and decreased musculotendinous stiffness [3,5].

As a tight iliotibial band may lead to patellofemoral pain syndrome in young adults, active stretching and strengthening exercise helps to reduce the tightness of iliotibial band and elongates the tight lateral structures of the knee joint [1]. The aim of the study was to find the effectiveness of iliotibial band stretching and strengthening exercises in patellofemoral pain syndrome. As vastus medialis muscle weakness becoming more common these days, which leads to shortening of lateral structures of the knee joint. A tight iliotibial band or lateral knee structure may cause maltracking of patella or lateral patellar tracking, which is one of the factors which leads to patellofemoral syndrome; hence, the purpose of this study was to find the whether stretching and strengthening iliotibial band in patellofemoral pain syndrome was significantly effective or not, in treating patellofemoral pain syndrome.

\section{METHODS}

Twenty patients were taken based on the inclusion and exclusion criteria; informed consent was obtained after explaining clearly about the iliotibial band stretching and strengthening exercises protocol. Institutional Ethical Committee approval was obtained before starting the study. Each patient was given 1 week intervention of IFT treatment and iliotibial band stretching and strengthening exercises.

The study was a quasi-experimental study and pre- and post-type. The study setting was SRM Medical College Hospital and Research Centre. Inclusion criteria of this study were patient diagnosed with patellofemoral pain syndrome, individuals' positive with Clarke's sign, both male and female, age group of 18-40 years, and visual analog scale (VAS) 3-7. Exclusion criteria of this study were current back, hip, or ankle pain, previous surgery or traumatic injury to back, hip, knee, or ankle, rheumatoid arthritis, medial and lateral meniscus injury, osteoporosis of patellofemoral joint, and osteoarthritis of knee.

Clarke's sign (patellar grind test) was performed to confirm patellofemoral pain syndrome. In this, the examiner presses down slightly proximal to the upper pole or base of the patella with the web of the hand as the patient lies relaxed with the knee extended pushing down on the patella directly. The patient was then asked to contract the quadriceps muscle while the examiner pushes down. If the test causes retropatellar pain and the patient cannot hold the contraction, the test was considered positive. The amount of pressure applied must be controlled carefully. Test should be performed on both affected and non-affected leg to find the difference [6].

The Ober's test was conducted on both legs of each patient $[1,7,4]$.

Position of the patient was side-lying. Position of the therapist was behind the patient. In side-lying, the lower leg was flexed to $45^{\circ}$ to maintain a neutral lumbar lordosis. Pelvis was stabilized with hand as necessary.

The upper leg knee was flexed to $90^{\circ}$ and leg was passively brought into abduction and extension.

An inclinometer was placed on the lateral thigh, just proximal to the lateral femoral condyle. The therapist lowered the leg into adduction, attempting to control for any visually observed unwanted hip rotation. The end point (angle of hip adduction) was deemed when no further adduction occurred, and the reading was taken from the inclinometer.

If the limb was horizontal, it was considered to be at $0^{\circ}$, if below horizontal (adducted), the angle was recorded as a positive number, and if above horizontal (abducted), the angle was recorded as a negative number.

An independent observer recorded all the readings. Each leg was measured on one occasion only.

For those patients, who had a negative reading were included in the study, were undergone 1 week duration treatment with IFT for pain relief and iliotibial band stretching and strengthening exercises. At the end of 1 week, again readings were taken with inclinometer while performing Ober's test.

\section{IFT treatment}

\section{Position of the patient was supine lying}

Four electrodes were placed around knee joint diagonally opposite one another so that beat frequency is produced. Beat frequency: $100 \mathrm{~Hz}$, base: $20 \mathrm{~Hz}$, spectrum: $80 \mathrm{~Hz}$, treatment time: 10 minutes, intensity: According to patient tolerance. IFT was given to each patient one time a day for 1 week duration [3]. Iliotibial band stretching and strengthening exercises were followed after this treatment.

\section{Iliotibial band active stretching exercises $[8,9]$}

In the first stretch, the patient should stand near wall to get support by one hand, and the affected or painful leg was crossed behind normal leg, and patient leaned toward normal side. The stretch should be held for 30 seconds; five repetitions were performed in one set. Three sets were performed at three different times a day for 1 -week duration.

In the second stretch, the patient was asked to cross painful leg behind the normal leg and lean toward the normal leg side. This stretch was best performed with arms over the head, creating a "bow" from ankle to hand on the affected leg. The stretch should be held for 30 seconds; five repetitions were performed in one set. Three sets were performed at three different times a day for 1 -week duration.

In the third stretch, the patient was asked to cross the painful leg behind normal leg, painful leg side arm above head, and lean toward normal side. The stretch should be held for 30 seconds; five repetitions were performed in one set. Three sets were performed at three different times a day for 1-week duration.

\section{Side lying hip abduction}

Patient was asked to lie on side, bend the knee of the normal leg while keeping the upper leg straight lift the painful leg again, isolate the motion to hip do not lift too high and keep spine stable. Focus on keeping painful leg in line with trunk and foot parallel to the ground. Ten repetitions in one set three different times a day for 1 week were performed.

\section{Side lying calm}

Patient was asked to lie on side with elastic band around the thighs just above the knees. Keeping feet together, the upper leg or painful leg was lifted off the lower leg or normal leg. Leg was lifted only as high as comfortable, and the patient was asked to not allow back to rotate. Patient was asked to keep spine stable throughout the exercise and isolate the motion to the hip. Ten to fifteen repetitions in one set and performed three different times a day.

\section{RESULTS}

To find out the effectiveness of iliotibial band stretching and strengthening exercises in patellofemoral pain syndrome, paired t-test was used; to asses changes in VAS score and angle of hip adduction from pre-test to post-test.

Since the $p$ value $(<0.001)$ is $<0.05$. There was statistically significant difference between pre- and post-test VAS scores among patients with patellofemoral pain syndrome. 
Since the $p$ value $(<0.001)$ is $<0.05$. There was a significant difference between pre- and post-test hip adduction scores among patient with patellofemoral pain syndrome.

\section{DISCUSSION}

The purpose of this study was to find the effect of iliotibial band stretching and strengthening exercises in patellofemoral pain syndrome. The results would agree with the evidence that iliotibial band stretching and strengthening exercises were effective in treating patients with patellofemoral pain syndrome. The result shows that iliotibial band stretching and strengthening exercises were statistically significant in treating patients with patellofemoral pain syndrome.

The patella articulates with the patellofemoral groove in the femur. Several forces act on the patella to provide stability and keep it tracking properly. A common misconception is that the patella only moves in an up and down direction. In fact, it also tilts and rotates, so there are various points of contact between the undersurface of the patella and the femur. Repetitive contact at any of these areas, sometimes combined with maltracking of the patella that is often not detectable by the naked eyes is the likely mechanism of patellofemoral pain syndrome $[2,10]$.

Patellofemoral pain syndrome is often classified as an overuse injury because bending the knee increases the pressure between the patella and its various point of contact with femur. Repeated weight-bearing impact may be a contributing factor, particularly in runners. Steps, hills, and uneven surfaces tend to exacerbate patellofemoral pain $[2,10]$

Once the patellofemoral pain syndrome has developed, even prolonged sitting can be painful (Moviegoers sign) because of the extra pressure between the patella and the femur during knee flexion. A large Q-angle is also a predisposing factor for patellofemoral pain [11]. Comparison of pre-test and post-test values of VAS score among patients with patellofemoral pain syndrome using paired t-test showed that there was a statistically significant difference with $t$ value 17.59 at $p<0.001$. Hence, the pain has reduced at the end of 1-week intervention of IFT and iliotibial band stretching and strengthening exercises.

The iliotibial band has a both dynamic and passive role at patellofemoral joint. Proximally, the iliotibial band attaches to the tensor fascia lata

Table 1: Comparison of pre- and post-test scores of VAS among patients with patellofemoral pain syndrome

\begin{tabular}{|c|c|c|c|c|c|}
\hline \multicolumn{2}{|l|}{ ??? } & \multicolumn{2}{|l|}{$\mathbf{n}$} & \multicolumn{2}{|c|}{ Mean \pm standard division } \\
\hline \multirow{2}{*}{\multicolumn{2}{|c|}{$\begin{array}{l}\text { Pre-VAS } \\
\text { Post-VAS }\end{array}$}} & \multicolumn{2}{|l|}{20} & \multicolumn{2}{|c|}{$5.675 \pm 1.150$} \\
\hline & & \multicolumn{2}{|l|}{20} & \multicolumn{2}{|c|}{$2.575 \pm 1.092$} \\
\hline \multicolumn{6}{|c|}{ VAS: Visual analog scale } \\
\hline & \multirow[t]{2}{*}{ t value } & \multirow[t]{2}{*}{ D.F } & \multirow[t]{2}{*}{ p value } & \multicolumn{2}{|c|}{ Confidence interval } \\
\hline & & & & Lower & Upper \\
\hline VAS Score & 17.5 & 19 & $<0.001$ & 2.731 & 3.469 \\
\hline
\end{tabular}

Table 2: Comparison of pre- and post-test scores of hip adduction angle among patients with patellofemoral pain syndrome

\begin{tabular}{|c|c|c|c|c|c|}
\hline & & $\mathbf{n}$ & \multicolumn{3}{|c|}{ Mean \pm standard deviation } \\
\hline Pre-hip_adduct & & 20 & \multirow{2}{*}{\multicolumn{2}{|c|}{$\begin{array}{l}-11 \pm 2.53 \\
-6.85 \pm 2.50\end{array}$}} & \\
\hline Post-hip_adduc & & 20 & & & \\
\hline & \multirow[t]{2}{*}{ t value } & \multirow[t]{2}{*}{ D.F } & \multirow[t]{2}{*}{ p value } & \multicolumn{2}{|c|}{ Confidence interval } \\
\hline & & & & Lower & Upper \\
\hline $\begin{array}{l}\text { Hip adduction } \\
\text { score }\end{array}$ & -11.61 & 19 & $<0.001$ & -4.9 & -3.4 \\
\hline
\end{tabular}

while distally, fibers from the iliotibial band interdigitate with vastus lateralis. Most of the lateral retinaculum arises from the iliotibial band; therefore, the iliotibial band indirectly provides lateral stabilization and acts as a passive restraint to medial patellar glide. A tight iliotibial band could lead to lateral patellar tracking, lateral patella tilt, and lateral patella compression. Using inclinometer to measure hip adduction using the Ober's test is a reliable method for the measurement of iliotibial band flexibility $[8,12]$.

Interferential therapy works on pain gait mechanism which reduces the pain perception by blocking the pain impulses by stimulating nociceptors, mechanoreceptor, and blocking the pain by pre-synaptic inhibition of the small diameter nociceptive nature; if large diameter afferent information is superimposed; then, the gate is closed to nociceptive traffic. If nociceptive information is allowed through the gate, then this traffic will continue up the lateral spinothalamic tract of the spinal cord to the thalamus, and from here to the cerebral cortex. As this stimulus passes through the brainstem, it may cause an interaction between the periaqueductal area of gray matter and the raphe nucleus in the mid-brain. These nuclei form part of the descending pain suppression system, and their descending neurons can release an endogenous opiate substance into the substantia gelatinosa at a spinal cord level. Beta-endorphin and enkephalin inhibit the release of pain-producing substance (p substance); it also causes inhibition of transmission in the nociceptive circuit synapse. The interferential effect produces a reduction in pain by closing the pain gate [13].

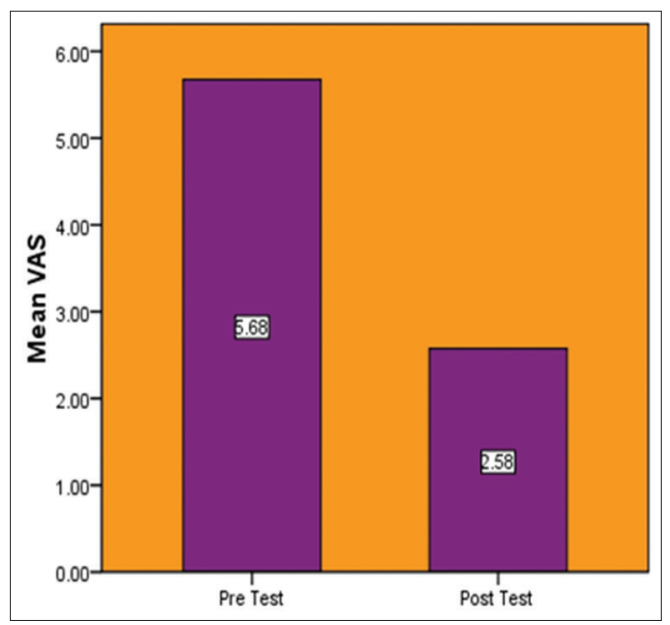

Graph 1: Comparison between pre- and post-test visual analog scale score among patients with patellofemoral pain syndrome

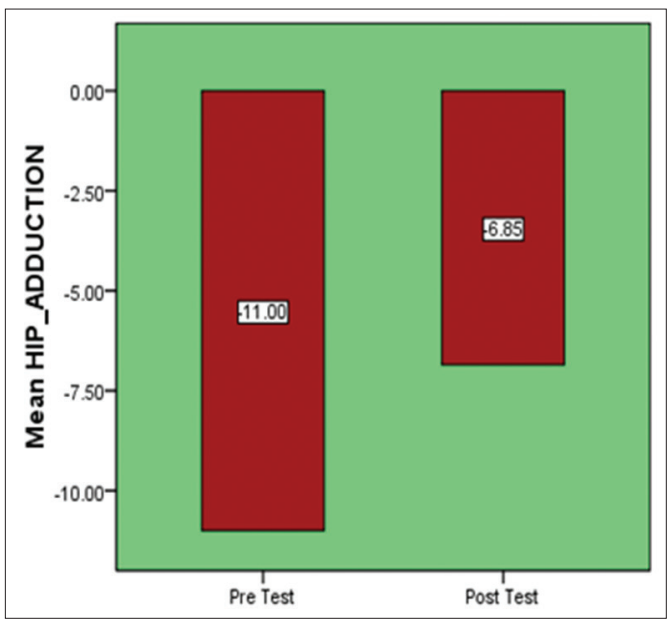

Graph 2: Comparison between pre- and post-angle of hip adduction among patients with patellofemoral pain syndrome 
Comparison of pre-test and post-test values of hip adduction score among patients with patellofemoral pain syndrome, using paired t-test showed that there was a statistically significant difference with $\mathrm{t}$-value -11.61 at $\mathrm{p}<0.001$. Hence, the hip adduction angle has increased at the end of 1-week intervention of iliotibial band stretching and strengthening exercises.

Patients presenting with patellofemoral pain syndrome had a tight iliotibial band in the painful knee. A tight iliotibial band is one of the factors which lead to patellofemoral pain syndrome. Poor control of medial hip rotation through gluteus maximus causes lateral tracking of patella during dynamic weight bearing. Hence, mobilizing tight lateral structures and stretching and strengthening iliotibial band with theraband can be useful in treating patellofemoral pain syndrome.

This study shows that there was a statically significant difference in pre- and post-test VAS score and in pre- and post-test angle of hip adduction after 1 week of intervention of IFT treatment and iliotibial band stretching and strengthening exercises. Thus, stretching and strengthening iliotibial band was effective in treating patellofemoral pain syndrome.

\section{CONCLUSION}

This study concluded that 1 week of stretching and strengthening iliotibial band exercises were effective in reducing pain and increasing hip adduction in patellofemoral pain syndrome. The limitations of this study were sample size was small, there was no control group in this study, all measurements for a given patient in the study were measured by the same individual, this study consisted of a short course of intervention over a mean period of 1 week. Further studies can be done with a greater body mass index to find the prevalence of patellofemoral pain syndrome in obese and overweight patients, find the effectiveness of iliotibial band stretching and strengthening exercises in patellofemoral pain syndrome with longer durations, and comparing the taping techniques and patellar mobilizations to treat patellofemoral pain syndrome.

\section{ACKNOWLEDGMENTS}

Authors express gratitude to those who supported to complete this research.

\section{REFERENCES}

1. Hudson ZL, Darthuy E. Iliotibial band tightness and patellofemoral pain syndrome: A case control study. Phys Ther Sport 2006;7(4):173.

2. Reese NB, Bandy WD. Use of an inclinometer to measure flexibility of the iliotibial band using the Ober test and the modified Ober test: Differences in magnitude and reliability of measurements. J Orthop Sports Phys Ther 2003;33(6):326-30.

3. De Domenico G. Pain relief with interferential therapy. Aust J Physiother 1982;28(3):14-8.

4. Melchione WE, Sullivan MS. Reliability of measurements obtained by use of an instrument designed to indirectly measure iliotibial band length. J Orthop Sports Phys Ther 1993;18(3):511-5.

5. Fredericson M, White JJ, Macmahon JM, Andriacchi TP. Quantitative analysis of the relative effectiveness of 3 iliotibial band stretches. Arch Phys Med Rehabil 2002;83(5):589-92.

6. Magee DJ. Orthopedic Physical Assessment. $3^{\text {rd }}$ ed. Philadelphia, PA: W.B. Saunders; 1997. p. 566.

7. Herrington L, Rivett N, Munro S. The relationship between patella position and length of the iliotibial band as assessed using Ober's test. Man Ther 2006;11(3):182-6.

8. Wang TG, Jan MH, Lin KH, Wang HK. Assessment of stretching of the iliotibial tract with Ober and modified Ober tests: An ultrasonographic study. Arch Phys Med Rehabil 2006;87(10):1407-11.

9. Brotzman SB. Clinical Orthopedic Rehabilitation. $2^{\text {nd }}$ ed. Philadelphia, PA: Mosby; 2003. p. 520.

10. Puniello MS. Iliotibial band tightness and medial patellar glide in patients with patellofemoral dysfunction. J Orthop Sports Phys Ther 1993;17(3):114-8.

11. Mark S, Juhn DO. Patellofemoral pain syndrome: A review and guidelines for treatment. Am Fam Physician 1999;60(7):2012-8.

12. Fredericson M, Cookingham CL, Chaudhari AM, Dowdell BC, Oestreicher N, Sahrmann SA. Hip abductor weakness in distance runners with iliotibial band syndrome. Clin J Sport Med 2000;10(3):169-75.

13. Melzack R, Wall PD. Pain mechanism: A new theory. Science 1965;150(3699):971-9. 